\title{
Elastic Characteristics of Digital Cores from Longmaxi Shale Using Lattice Spring Models
}

\author{
Ning Liu ${ }^{1}$ and Li-Yun $\mathrm{Fu}^{2}$,* \\ ${ }^{1}$ Key Laboratory of Earth and Planetary Physics, Institute of Geology and \\ Geophysics, Chinese Academy of Sciences, Beijing 100029, China. \\ 2 School of Geosciences, China University of Petroleum (East China), Qingdao 266580, \\ Shandong, China.
}

Received 31 March 2018; Accepted (in revised version) 31 October 2018

\begin{abstract}
Effective medium methods for the attribution of micro-structures to macro elastic properties of shales are important for the prediction of sweet spots in the shalegas production. With X-ray micro-computed tomography (XMCT), the micro-structures of shale core samples from Longmaxi Formation are visualized and characterized by $3 \mathrm{D}$ digital images. As an efficient alternative to conventional effective medium methods for estimating elastic properties, we propose a consistent workflow of lattice spring modeling (LSM) to emulate the digital cores using three types of lattices. Particular attention is paid to investigate the effective Young's moduli, Poisson's ratios, and preferred orientations, by uniaxial compression tests along two directions. Within elastic deformation, the impact of lattice arrangements on the anisotropy is even more than those of stress disturbances and micro-structural features. Compared with analytical approximations and theoretical predictions, the LSM numerical scheme shows general applicability for heterogeneous porous rocks.
\end{abstract}

AMS subject classifications: 37K60, 65C20, 74B05, 74E10

Key words: Elastic characteristics, lattice spring model (LSM), X-ray micro-computed tomography (XMCT), digital cores.

\section{Introduction}

Shale gas is a kind of unconventional natural gas that is found trapped within the shale formations. In China, Longmaxi Formation at Sichuan Basin, due to its great deposition thickness, stable distributions and rich organic contents, is currently one of the most important stratigraphic horizons for shale gas exploration and exploitation. Knowledge on

${ }^{*}$ Corresponding author. Email addresses: lfu@mail.iggcas.ac.cn (L.-Y. Fu), nicolaliu@buaa.edu.cn (N. Liu) 
the characteristics of elastic properties, especially those related to TOC (total organic carbon), maturity, reservoir thickness, mineral composition, brittleness, permeability, porosity and pore pressure [1], is imperative for sweet spot prediction. Rather than experimental measurement and theoretical prediction, numerical simulation based on digital cores is an efficient alternative to investigate the dependence of shale elasticity on mineral compositions and micro-structures. Thus, it is fundamental to reconstruct petrophysically the geological structure of shale reservoirs.

For realistic modeling of the shale gas reservoirs, structural characteristics, natural fracture system, multi-fractured horizontal well, and the like, should be taken into account [2]. Currently, the micro-structures can be captured at micrometer resolution by imaging facilities such as scanning electron microscopy (SEM) and X-ray computed micro-tomography $(\mu \mathrm{CT})$, hopefully enabling the numerical investigation for the relation between micro-structures and the physical or mechanical properties of shales. Based on these high-resolution imaging techniques, howbeit several publications have reported numerical simulations of mechanical and seismic properties of geo-materials mainly implemented by finite difference methods (FDM) and finite element methods (FEM) [1]. Arns et al. [3] used FEM to obtain the elastic properties of Fontainebleau sandstone, which could agree with experimental measurements over a wide range of porosity. With higher resolution representation of complex micro-structural geometries, a deeper understanding of rock properties could be attained, e.g., elastic and transport properties $[4,5]$, electrical properties [6], pore fluid properties [7] and reservoir characteristics [8]. Zhang et al. attempted to derive the dependence of elastic properties on porosity and kerogen by FEM and described the micro-structural, lithological and petrophysical characteristics of the Longmaxi shale core [1].

Such studies principally are based on the continuum theories, yet it's more suitable to regard the rocks as granular media, which exhibit varying degrees of anisotropy (orientation dependence) caused by their crystalline structure, stress orientations, shape, and configuration. That's because the spatial heterogeneity and material anisotropy strongly influence crack patterns and effective fracture toughness, particularly seismic prospecting [9]. Crampin in his work ever mentioned that the coupling effects among the three body waves $\left(\mathrm{qP}, \mathrm{qS}_{1}\right.$, and $\left.\mathrm{qS}_{2}\right)$ motions may be sensitive even to quite weak anisotropy [10]. Therefore, discontinuum based methods which could account for granular texture, particle-scale kinematics and force transmission [11], like molecular dynamics (MD) and discrete element method (DEM), were proposed and developed for granular materials modeling. Hagenmuller [12] acquired DEM models according to the voxel (for 3D)/pixel (for 2D) coordinates, in order to study the rapid and large deformations of granular materials. Even though this kind of grain arrangement is one of the most convenient way to model discontinuum media, it may cause artificial anisotropy [13]. So as to avoid the anisotropic effects linked to regular packings, Harthong et al. [14] generated polydisperse packings to emulate rock masses, using the softer components [15] or discrete fracture networks (DFNs) [16] to represent the local heterogeneity and lamination. To some extent, despite the anisotropic nature of shale could be characterized, the 
impacts caused by multiphase minerals are neglected. Moreover, as for shale modeling, to the best knowledge of the authors, the correlated studies are quite scarce with limited technical details $[1,17]$.

Triggered by this current research status and the signification of shale modeling, a lattice model (LM) is applied here. Unlike the numerical approaches based on continuum assumptions that may barely handle large-scale fracturing, complex discontinuity simulations, or microscopic mechanisms [13], without the limitations of scale features and complex potential functions from discontinuum based methods (MD and DEM, etc.), LM scheme bears obvious advantages over others. At first, LM approach is flexible to model both granular media and continuum systems [18], whose scale characteristics of specimens may be extended by a coarser lattice idea [19]. Secondly, the singularity issues involved in the stress concentration around crack tips might be avoided. Additionally, this numerical strategy may have the easiest access to crack onset and propagation simulations, or cross-scale modeling for rock physics.

As a result, LMs have been proved to be sufficiently general for applying to systems ranging from metals, composites, ceramics and polymers to functionally graded and granular materials [18]. Due to the discrete nature of soil, rocks and the like, LMs are expansively used in the field of geophysics. As early as 1941, Hrennikoff developed the lattice spring model (LSM) to study the mechanical responses of solids with a fixed Poisson's ratio [19]. Based on this concept, in 1978, Nayfeh and Hefzy proposed a regular truss-like lattice model to calculate the effective mechanical properties [20]. Then, this approach was utilized to investigate distributed disorder influence [21], stress concentrations and toughness increases [22]. Also, other mechanical and acoustic behaviors could be studied by this methodology, eg. tectonic processes [23], earthquake dynamics [24], wave propagation [25], and earthquake generation and spread [26]; the fracture of elastic foundations on soft sand beds [27]; the generation of compaction bands in high-porosity sedimentary rock [28]; the scale effect in rock dowels [29]; the determination of static and dynamic fracture mechanics parameters and crack growth simulation [30]. Additionally, in order to extend the method into other fields besides geophysics and to avoid the Poisson's ratio limitation from conventional LMs, some other advanced models were proposed as well, like the Born spring model, the beam element model, the multibody shear spring, the nonlocal potential and the fourth-dimensional LSM [31].

While, the objective of this work is to develop a surrogate and potential approach regarding the shale core modeling with simple implementation and present a consistent simulation procedure with set-up details. Meanwhile, to attain complementary insights and an in-depth understanding of geological microstructures for the Longmaxi shale core, X-ray micro-CT techniques are employed. Some factors, like lattice arrangements, micro-structures and stress fields, that may attribute to anisotropic elasticity features are quantitatively compared and analyzed by bounding methods and a modified fabric tensor, accordingly. Based on these goals, the remaining parts of this paper are organized as follows.

The imaging means, composition identification, and evaluation for elastic parame- 
ters are outlined in Section 2. Subsequently, modeling algorithm of the adopted lattice models, including basic concepts, modeling procedure, the relations between inputs and effective elastic properties of lattice unit cells, and fabric tensors are expressed in Section 3. In Section 4, the numerical data of uniaxial compression tests are presented, covering comparisons with theoretical values to validate the model and to calibrate material parameters, prediction for effective elastic properties and evaluation of various bounding methods, and anisotropy characterization from the viewpoint of lattice orientations, inherent micro-structural features, and stress influences. Then, the predictive capability of this numerical scheme is discussed in Section 5. Finally, conclusions and future work are underlined in Section 6.

\section{Digital core imaging and elastic properties}

A Longmaxi shale core is scanned by $\mathrm{X}$-ray micro-computed tomography (XMCT) with a proper resolution to obtain a digital core image. Otsu's method is used to segment the digital core image according to the absorption coefficients of various constituents. We introduce several effective medium theories to estimate the specimen's elastic constants.

\subsection{Digital core imaging}

XMCT scanning of the Longmaxi shale core with a diameter of $800 \mu \mathrm{m}$ and a length of 3 $\mathrm{mm}$ is performed at Shanghai Synchrotron Radiation Facility (SSRF) using a third generation synchrotron radiation light source. For reconstructed precision improvement, the ordered subset expectation maximization (OSEM) algorithm is introduced. Figs. 1 illustrate the 3D model from three views, which is generated by 1080 projections at micrometer resolution with a $3.5 \mathrm{~s}$ exposure time. To capture mineral components and micro-structures of the sample more precise, we extract a cube with a size of $390 \times 390 \times 455 \mu \mathrm{m}^{3}$. The objective 3D digital image in a resolution of $0.65 \mu \mathrm{m}$, consists of $600 \times 600 \times 700$ voxels. Fig. 2 displays the resulting digital image (8-bit) converted from the original reconstructed image (32-bit), with the color scale ranging from 0 to 255 based on the X-ray absorption coefficients.

\subsection{Identification of constituents}

Shales consist of diverse minerals-detrital, clay, and mental minerals-, with irregular pores. The XMCT technique enables the possibility to visualize and characterize those minerals in digital cores, according to the X-ray reflectivity of different constituents. In other words, gray scale intensities change in the brightness of X-ray images reflect the chemical and optical properties of different minerals. For example, pyrite has a high absorption coefficient of X-ray than other minerals, leading to greater brightness in the image, whereas kaolinite, montmorillonite, and quartz have lower values. The absorption coefficients of those common minerals are reported in Table 1 [32]. We see that some 

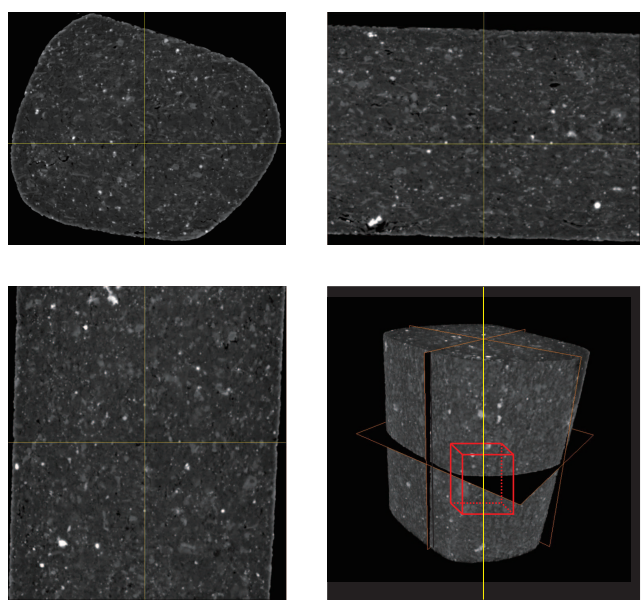

Figure 1: Three views of the original 3D model with the scanned cube.
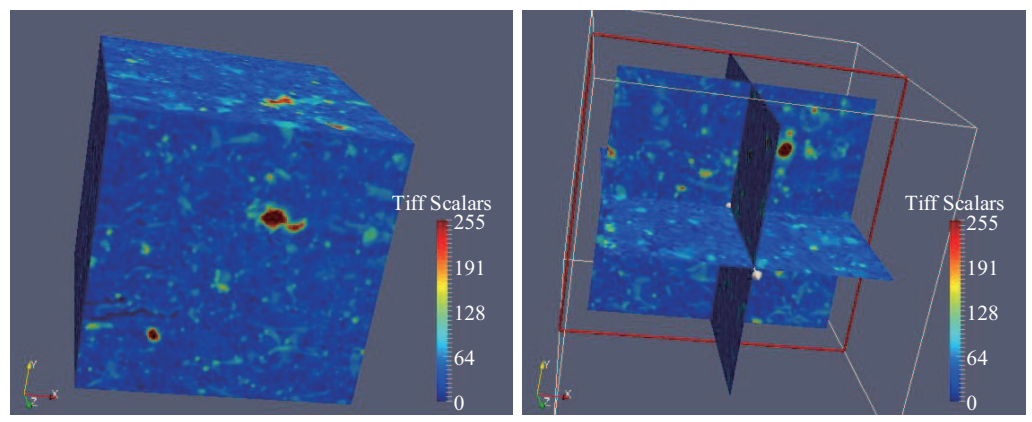

Figure 2: Reconstructed 3D image (left) with its 2D slices (right) in the $X Y, X Z$, and $Y Z$ planes.

of those minerals share similar values and could be classified into the same category of mineral components.

The 3D grayscale data is segmented into a few binary images using Otsu's method in terms of absorption coefficients listed in Table 1. From Fig. 3, see that Group 1 takes the majority of grayscale values. Pyrite just accounts for a small proportion, implying the Longmaxi shale might be deposited in a strong reducing sedimentary environment. Fig. 4(a) shows the percentage of three mineral groups and pores in the 3D image. We see that the Longmaxi shale core contains Mineral Group 1 up to $78.33 \%$ with a high percentage of detrital minerals. The other two mineral groups and pores hold $16.53 \%$, $0.64 \%$, and $4.51 \%$, respectively.

Table 1: Absorption coefficients of $\mathrm{X}$-ray with $20 \mathrm{keV}$ energy for common minerals in the shale sample [1].

\begin{tabular}{||llllllllll||}
\hline Mineral & Kln & Mnt & Qtz & Ill & Kfs & Dol & Gp & Cal & Py \\
\hline Coefficient $\left(\mathrm{cm}^{-1}\right)$ & 5.7 & 6.4 & 6.5 & 8.9 & 10.3 & 10.5 & 13.1 & 15.0 & 76.7 \\
\hline
\end{tabular}




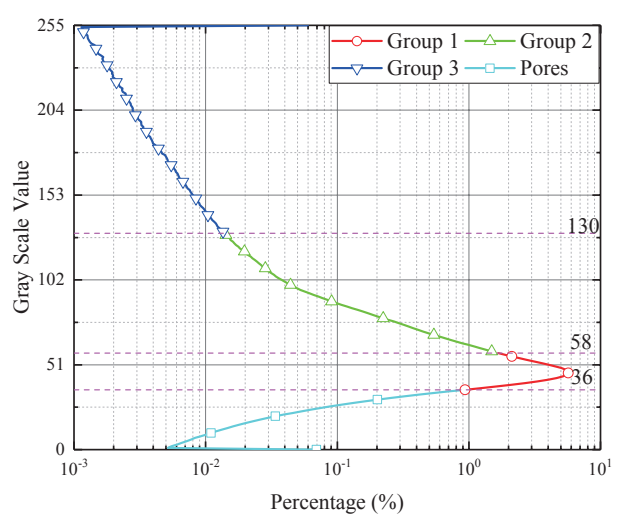

Figure 3: Percentage of the 3D image voxel at every grayscale value in the range of 0 to 255: Group 1 (kaolinite, quartz, and montmorillonite) in 37-58, Group 2 (illite, K-feldspar, calcite, dolomite, and gypsum) in 59-130, Group 3 (pyrite) in 131-255, and pores in $0-36$.

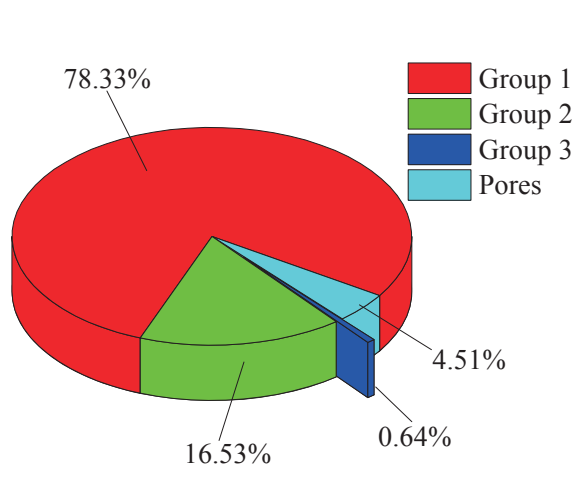

(a)

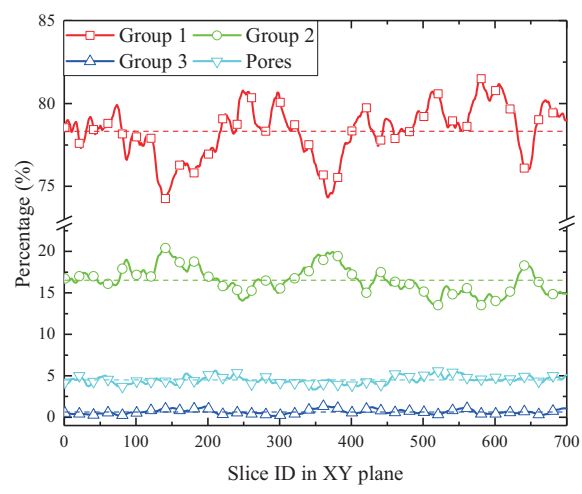

(b)

Figure 4: Composition statistics of the Longmaxi shale core: (a) the proportions of mineral groups and pores; (b) the composition distributions of 700 slices in $\mathrm{Z}$ direction.

Therefore, the Longmaxi shale seems brittle and tight, with its permeability and connectivity sensitive to stress fields. Fig. 4(b) shows the percentage of three mineral groups and pores for $7002 \mathrm{D}$ slices in the $X Y$ planes. All the values for each group fluctuate around the average line. That is because every slice along the $\mathrm{Z}$ direction displays approximately the similar mineral distribution. Hence for simplicity, we can emulate the Longmaxi shale sample just from one of the 2D slices in the XY planes, as illustrated in Fig. 5. Based on these 2D cross-section slices, we can identify and separate mineral phases and porous structures by image segmentation. In the binary images, the black pixels present the mineral groups and pores, with their percentages of $76.17 \%$ (Mineral Group 1), 19.41\% (Mineral Group 2), 1.06\% (Mineral Group 3), and 3.36\% (pores), correspondingly. 

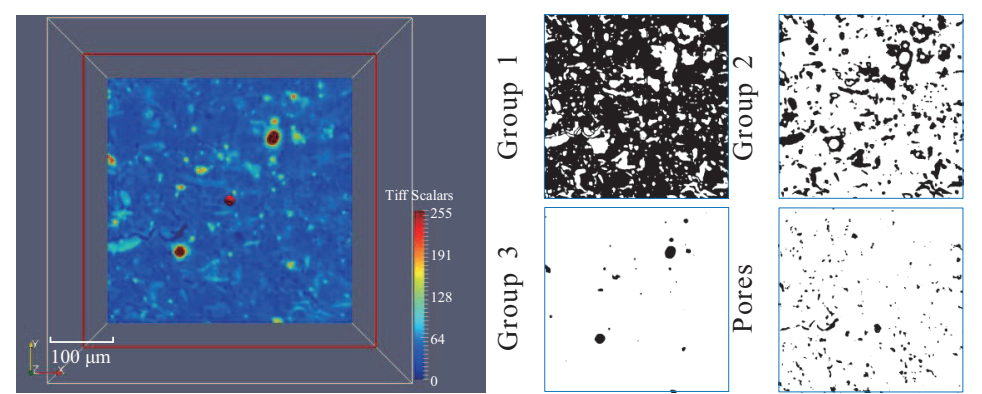

Figure 5: Composition identification of the digital core: one of the 2D slices along the Z direction (left); binary images of three mineral groups and porous structures (right).

\subsection{Estimation of effective elastic properties}

As described in Fig. 5, Groups 1 and 2 are the fundamental constituents in the Longmaxi shale core. We can estimate the effective elastic modulus of each mineral group for numerical simulations. The upper-and-lower bound method usually act as a powerful and robust tool to attain the limit on the material parameters, and are used in the current study.

Because of the complexity of micro-structures, the geometric details of grains and pores are rarely taken into account. Solely based on phase volume fractions, the VoigtReuss model presents upper and lower bounds on the effective linear elastic moduli of multi-phase composites. It can be constructed from the principle of energy minimum at the equilibrium state. In the field of rock physics, the Voigt-Reuss bounds have been popularly used, written as:

$$
\bar{M}^{\alpha}=f_{1} M_{1}^{\alpha}+f_{2} M_{2}^{\alpha}+\cdots+f_{i} M_{i}^{\alpha}+\cdots,
$$

where $\alpha=1$ or $\alpha=-1$ for Voigt or Reuss bounds, correspondingly. $\bar{M}, M_{i}$ and $f_{i}$ stand for the effective modulus, and the individual modulus and volume fraction of the $i^{\text {th }}$ constituent. Then, the Voigt-Reuss-Hill average is applied, since sometimes an estimate of the actual value is taken as the average of the two:

$$
\bar{M}=\frac{M_{\mathrm{V}}+M_{\mathrm{R}}}{2},
$$

where $M_{\mathrm{V}}$ and $M_{\mathrm{R}}$ represent the effective moduli based on Voigt and Reuss bounds. A more accurate estimation of bounding methods can be obtained for two-phase composites based on variational principle, which has been developed for multiphase media [33] and expressed in a general form

$$
\begin{aligned}
& K^{\mathrm{HS}+}=\left[\sum_{i=1}^{N} \frac{f_{i}}{K_{i}+z_{K}^{\mathrm{HS}+}}\right]^{-1}-z_{K}^{\mathrm{HS}+}, \quad z_{K}^{\mathrm{HS}+}=\frac{2(d-1)}{d} \mu_{\max } ; \\
& K^{\mathrm{HS}-}=\left[\sum_{i=1}^{N} \frac{f_{i}}{K_{i}+z_{K}^{\mathrm{HS}-}}\right]^{-1}-z_{K}^{\mathrm{HS}-}, \quad z_{K}^{\mathrm{HS}-}=\frac{2(d-1)}{d} \mu_{\min } ;
\end{aligned}
$$




$$
\begin{aligned}
& \mu^{\mathrm{HS}+}=\left[\sum_{i=1}^{N} \frac{f_{i}}{\mu_{i}+z_{\mu}^{\mathrm{HS}+}}\right]^{-1}-z_{\mu}^{\mathrm{HS}+}, \quad \mu^{\mathrm{HS}-}=\left[\sum_{i=1}^{N} \frac{f_{i}}{\mu_{i}+z_{\mu}^{\mathrm{HS}-}}\right]^{-1}-z_{\mu}^{\mathrm{HS}-} ; \\
& z_{\mu}^{\mathrm{HS}+}=\frac{\mu_{\max }\left(9 K_{\max }+8 \mu_{\max }\right)}{6 K_{\max }+12 \mu_{\max }}, \quad z_{\mu}^{\mathrm{HS}-}=\frac{\mu_{\min }\left(9 K_{\min }+8 \mu_{\min }\right)}{6 K_{\min }+12 \mu_{\min }} \quad(d=3), \\
& z_{\mu}^{\mathrm{HS}+}=\frac{K_{\max } \mu_{\max }}{K_{\max }+2 \mu_{\max }}, \quad z_{\mu}^{\mathrm{HS}-}=\frac{K_{\min } \mu_{\min }}{K_{\min }+2 \mu_{\min }} \quad(d=2) .
\end{aligned}
$$

Here, $K$ and $\mu$ are bulk and shear moduli; the superscripts HS+ and HS- symbol the upper and lower values of the Hashin-Shtrikman-Walpole bounds, respectively; the subscript $\max (\mathrm{min})$ represents the maximal (minimal) value; and $d=2$ or $d=3$ is the spatial dimension.

The contents and material constants of each constituent in Groups 1 and 2 are listed in Table 2, computed by Eqs. (2.1)-(2.6) in terms of X-ray diffractions [1,34]. In the table, $\rho$ and $f$ denote the density and the percentage of each phase, respectively. Reasonable assumptions are included, i.e., kaolinite/montmorillonite in Mineral Group 1 and illite/gypsum in Mineral Group 2 take the lower and higher elastic values for the clay aggregates [35], respectively. Table 3 summaries the effective bulk and shear moduli calculated by the Voigt-Reuss model, the Voigt-Reuss-Hill average, and the Hashin-Shtrikman

\begin{tabular}{|c|c|c|c|c|c|c|}
\hline \multirow[t]{2}{*}{ Parameter } & \multicolumn{2}{|c|}{ Group 1} & \multicolumn{4}{|c|}{ Group 2} \\
\hline & Qtz & Kln,Mnt & Kfs & Dol & Cal & $\mathrm{Ill}, \mathrm{Gp}$ \\
\hline$\rho\left(\mathrm{g} / \mathrm{cm}^{3}\right)$ & 2.65 & 2.13 & 2.75 & 2.87 & 2.71 & 2.83 \\
\hline$f(\%)$ & 48.20 & 51.80 & 34.00 & 27.30 & 23.70 & 15.00 \\
\hline$K(\mathrm{GPa})$ & 36.40 & 6.00 & 92.00 & 95.00 & 77.00 & 12.00 \\
\hline$\mu(\mathrm{GPa})$ & 31.14 & 4.00 & 41.00 & 45.00 & 32.00 & 6.00 \\
\hline
\end{tabular}
bounds, as well as other indispensable material parameters.

Table 2: Material constants of the constituents in Groups 1 and $2[1,34]$.

\begin{tabular}{|c|c|c|c|c|c|c|c|c|}
\hline \multirow[t]{2}{*}{ ID } & \multirow{2}{*}{$\begin{array}{l}\text { Volume } \\
(\%)\end{array}$} & \multirow{2}{*}{$\begin{array}{l}\text { Density } \\
\left(\mathrm{g} / \mathrm{cm}^{3}\right)\end{array}$} & \multirow{2}{*}{$\begin{array}{l}\text { Modulus } \\
(\mathrm{GPa})\end{array}$} & \multicolumn{5}{|c|}{ Bounding Methods } \\
\hline & & & & Voigt & Reuss & V-R-H & $\mathrm{H}_{-} \mathrm{S}^{+}$ & $\mathrm{H}^{-\mathrm{S}^{-}}$ \\
\hline \multirow[t]{2}{*}{1} & 76.17 & 2.38 & $K$ & 20.65 & 10.04 & 15.35 & 17.01 & 12.13 \\
\hline & & & $\mu$ & 17.08 & 6.90 & 11.99 & 13.28 & 8.78 \\
\hline \multirow[t]{2}{*}{2} & 19.41 & 2.79 & $K$ & 59.02 & 52.44 & 55.73 & 68.02 & 53.20 \\
\hline & & & $\mu$ & 34.71 & 25.41 & 30.06 & 32.08 & 26.16 \\
\hline \multirow[t]{2}{*}{3} & 1.06 & 4.93 & $K$ & 147.40 & 147.40 & 147.40 & 147.40 & 147.40 \\
\hline & & & $\mu$ & 132.50 & 132.50 & 132.50 & 132.50 & 132.50 \\
\hline
\end{tabular}

Table 3: Effective elastic properties of mineral groups. 


\section{Lattice modeling methods}

\subsection{Theoretical foundations}

Lattice modeling (LM) methods stem from the atomic lattice structure of materials, in which the object is discretized into mass nodes, or rheological elements, connecting with springs or beams [18]. Specifically in this paper, the shale core is represented by a lattice spring model (LSM) whose nodes interact with each other through normal and shear springs. Assuming that the springs behave in a linear elastic way, the interaction forces between two lattice nodes $i$ and $j$, including normal force $F_{\mathrm{n}}^{i j}$ and tangential force $F_{\mathrm{s}}^{i j}$, can be written as

$$
F_{\mathrm{n}}^{i j}=k_{\mathrm{n}} u_{\mathrm{n}}^{i j}, \quad F_{\mathrm{s}}^{i j}=k_{\mathrm{s}} u_{\mathrm{s}}^{i j},
$$

where $k_{\mathrm{n}}, k_{\mathrm{s}}, u_{\mathrm{n}}^{i j}$ and $u_{\mathrm{s}}^{i j}$ are the normal and shear spring stiffness and the relative distances in normal and tangential directions, correspondingly.

In this lattice model, every nodal force is accumulated from the interaction. Verlet leapfrog scheme, commonly used in molecular dynamics (MD), is introduced to integrate individual motion equations related to every node. Based on the algorithm, a set of vectors, coordinate $\mathbf{x}_{i}$, velocity $\mathbf{v}_{i}$, and acceleration $\mathbf{a}_{i}$ of the $i^{t \text { th }}$ node, the acceleration at $t$, $\mathbf{a}_{i}{ }^{(t)}$, and the velocity at $t+\frac{\Delta t}{2}, \mathbf{v}_{i}{ }^{\left(t+\frac{\Delta t}{2}\right)}$, are formulated by $\mathbf{x}_{i}^{(t)}, \mathbf{v}_{i}{ }^{\left(t-\frac{\Delta t}{2}\right)}$ and $\mathbf{a}_{i}{ }^{(t-\Delta t)}$ as

$$
\mathbf{a}_{i}{ }^{(t)}=\frac{\sum \mathbf{F}_{i}{ }^{(t)} \Delta t}{m_{i}}, \quad \mathbf{v}_{i}^{\left(t+\frac{\Delta t}{2}\right)}=\mathbf{v}_{i}^{\left(t-\frac{\Delta t}{2}\right)}+\mathbf{a}_{i} \Delta t, \quad \mathbf{x}_{i}^{(t+\Delta t)}=\mathbf{x}_{i}^{(t-\Delta t)}+\mathbf{v}_{i}^{\left(t+\frac{\Delta t}{2}\right)} \Delta t,
$$

where $\mathbf{F}_{i}$ is calculated from Eq. (3.1). A limit, called the critical time step $\Delta t_{\mathrm{cr}}$, is imposed on the time step $\Delta t$ to ensure the stability for this explicit integration algorithm, which is expressed by

$$
\Delta t \leq \Delta t_{\mathrm{cr}}, \quad \Delta t_{\mathrm{cr}}=\frac{2}{\omega_{\max }}
$$

where $\omega_{\max }$ is the largest eigenfrequency within the model. In order to obtain quasi-static solutions, a non-viscous damping is used to dissipate kinetic energy [36]. The damped force $\Delta \mathbf{F}_{\text {damped }}$ of node $i$ may be written as follows,

$$
\frac{(\Delta \mathbf{F})_{\text {damped }}^{i}}{\mathbf{F}_{i}}=-\xi \operatorname{sgn}\left(\mathbf{v}_{i}^{\left(t-\frac{\Delta t}{2}\right)}+\frac{\mathbf{a}_{i} \Delta t}{2}\right) .
$$

\subsection{Simulation procedure}

LM is different from the numerical methods based on continuum theories, the discretization may affect not only the accuracy but also the anisotropy. Fig. 6 gives three basic types of lattice arrangements: Model 1 (basic square lattices), Model 2 (nonlocal square lattices), and Model 3 (regular triangle lattices). The lattice nodes of Models 1 and 2 are generated based on the coordinates of the image pixels, which is a quite simple and common way. 


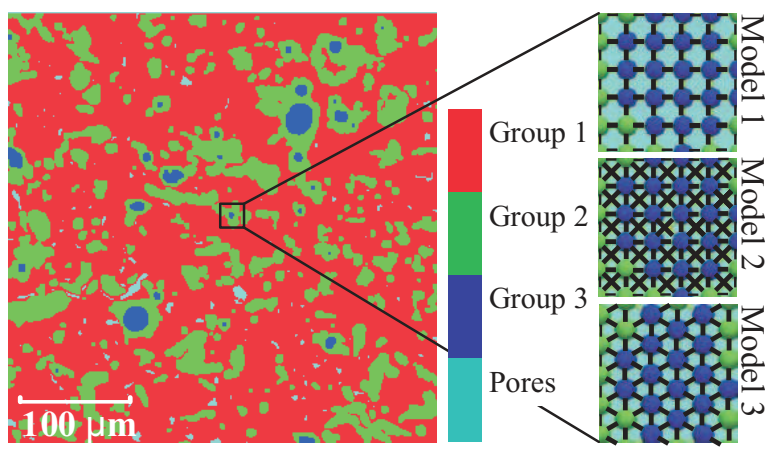

Figure 6: Three lattice arrangements for computation of the Longmaxi shale core.

For Model 3, the coordinates of mass nodes are set by the approximate zones of the pixels within the digital core image as shown in Fig. 5, which differs from the traditional meshing means. After initial positions are pre-determined, the nodes are connected by normal and shear springs within given lattice spacings. It is worth mentioning that the nonlocal model (Model 2) contains two force structures with: Structure I (a basic square network as same as Model 1) and Structure II (a square network with long-range interactions whose spacings are $\sqrt{2}$ times those of Models 1 and 3. The forces acting on every node are calculated by Eq. (3.1), and then the position of every node could be updated on every time step by Eq. (3.2). More implementation details can be found in our previous work [37] as well.

\subsection{Parameter identification}

To establish the relation between lattice parameters and macroscopic material properties, the conservation law of strain energy is introduced [18],

$$
U_{\text {cell }}=U_{\text {continuum }}, \quad \omega_{\text {cell }}=\frac{U_{\text {cell }}}{A_{\text {cell }}},
$$

where $U_{\text {cell }}$ and $\omega_{\text {cell }}$ are the energy and energy density of a unit cell, and $U_{\text {continuum }}$ is the counterpart in continuum theories. Thereby, the elastic moduli could be calculated by:

$$
C_{i j k l}=\frac{\partial^{2} \omega_{\text {cell }}}{\partial{ }^{\prime \prime}{ }_{i j}{ }_{k l}}
$$

The fourth-rank elasticity tensors could be reduced to second-rank ones using Voigt notation. From Table 4, the relations between material elastic properties and lattice inputs are calculated by Eqs. (3.5) and (3.6). We estimate the effective elastic constants of each mineral group by the V-R-H average. Then, all the spring parameters for Model 3 can be calculated by substituting the elastic properties (listed in Table 3) into the formulas shown in Table 4. 
Table 4: Relationships between spring stiffness and Lamé parameters [18].

\begin{tabular}{||llll||}
\hline Parameters & Model 1 & Model 2 & Model 3 \\
\hline$\lambda$ & $0\left(\frac{1}{2}\left(k_{\mathrm{n}}-k_{\mathrm{s}}\right)\right)$ & $-\frac{1}{2} k_{\mathrm{s}}\left(\frac{1}{2}\left(k_{\mathrm{n}}-k_{\mathrm{s}}\right)\right)$ & $\frac{\sqrt{3}}{8}\left(k_{\mathrm{n}}-k_{\mathrm{s}}\right)$ \\
$\mu$ & $\frac{1}{2} k_{\mathrm{s}}$ & $\frac{1}{2}\left(k_{\mathrm{n}}+k_{\mathrm{s}}\right)$ & $\frac{\sqrt{3}}{8}\left(k_{\mathrm{n}}+k_{\mathrm{s}}\right)$ \\
\hline
\end{tabular}

\subsection{Fabric tensors}

Shale is known as a kind of strongly anisotropic medium. Usually, this anisotropy is stereotypically characterized by directional features of granular contacts or micro-structures, which could be termed as fabric tensors [11]. Ken-Ichi [38] postulates mathematically three types of fabric tensors. Zysset and Curnier [39] formulates a fourth-rank representation as an alternative for micro-structural characteristics. Generally, the directional distribution function $f$ can be expanded in a convergent Fourier series as follows [40]:

$$
f(\mathbf{N})=G^{(0)} \cdot 1+\mathbf{G}^{(2)}: \mathbf{F}^{(2)}(\mathbf{N})+\mathbf{G}^{(4)}:: \mathbf{F}^{(4)}(\mathbf{N})+\cdots,
$$

where $\mathbf{N}$ is the fabric tensor of the first kind. The zeroth-rank, second-rank, fourth-rank and eighth-rank fabric tensors are given with $i, j, k, l, m, n, p, q=1,2$ for 2D problems by

$$
\begin{aligned}
& N^{(0)}=1, \quad N_{i j}{ }^{(2)}=\sum_{\alpha=1}^{N_{\mathrm{c}}} \frac{1}{N_{\mathrm{c}}} \mathbf{n}_{i}^{(\alpha)} \mathbf{n}_{j}^{(\alpha)}, \quad N_{i j k l}{ }^{(4)}=\sum_{\alpha=1}^{N_{\mathrm{c}}} \frac{1}{N_{\mathrm{c}}}\left(\mathbf{n}_{i} \mathbf{n}_{j} \mathbf{n}_{k} \mathbf{n}_{l}\right)^{(\alpha)}, \\
& N_{i j k l m n p q}{ }^{\left({ }^{(\alpha)}\right.}=\sum_{\alpha=1}^{N_{\mathrm{c}}} \frac{1}{N_{\mathrm{c}}}\left(\mathbf{n}_{i} \mathbf{n}_{j} \mathbf{n}_{k} \mathbf{n}_{l} \mathbf{n}_{m} \mathbf{n}_{n} \mathbf{n}_{p} \mathbf{n}_{q}\right)^{(\alpha)},
\end{aligned}
$$

where $N_{\mathrm{c}}$ and $\mathbf{n}$ are the number and normal vector of each interaction in the domain. The superscript $\alpha$ means the $\alpha^{\text {th }}$ interaction in the assembly.

\section{Numerical results}

In this section, we aim at the investigation of effective elasticity and anisotropy in the Longmaxi shale core based on the LSM numerical simulation of several uniaxial compression tests. The numerical implementation is verified by performing numerical tests using regular triangle lattices and comparing with the theoretical solutions of Young's modulus $E$ and Poisson's ratio $v$ calculated from Table 4 . The applicability of the bounding methods to shale core simulations is evaluated. The major factors that may cause anisotropy are discussed, such as lattice arrangements, mineral and porous features, and stress fields.

\subsection{Validation by theoretical solutions}

Table 5 shows an excellent agreement for the Young's moduli between the numerical and theoretical results, but with relatively larger errors for the Poisson's ratios. We can 
Table 5: The assessment of simulation errors of each group.

\begin{tabular}{||lllllll||}
\hline Group ID & \multicolumn{3}{l||}{ Young's modulus E (GPa) } & \multicolumn{4}{l||}{ Poisson's ratio $v$} \\
\cline { 2 - 7 } & Theoretical & Numerical & Error & Theoretical & Numerical & Error \\
\hline 1 & 26.93 & 26.90 & $1.01 \%$ & 0.12 & 0.11 & $8.11 \%$ \\
2 & 78.11 & 70.40 & $9.87 \%$ & 0.30 & 0.29 & $1.91 \%$ \\
3 & 279.11 & 302.00 & $8.20 \%$ & 0.05 & 0.05 & $3.92 \%$ \\
\hline
\end{tabular}

see the errors remain within a reasonable range (less than 10\%), indicating the general applicability of LSM modeling in this study.

\subsection{Elasticity compared with bounding methods}

In this study, we emulate the 2D Longmaxi digital core by uniaxial stress tests in the $X$ and $Y$ directions, with the corresponding Young's moduli and Poisson's ratios written as $E_{x x}, E_{y y}, v_{x y}$ and $v_{y x}$. Taking Model 1 for instance, Model 1-1 and Model 1-2 denote the uniaxial compression tests of Model 1 along the $X$ and $Y$ directions, respectively. Even though the breakage-and-creation mechanism is not considered, we still regard it as an interaction increase (decrease), when the distance between two lattice nodes is smaller (bigger) than the original value. The kind of number variation of the lattice interactions quantified by $\frac{N_{\mathrm{c}}-N_{\mathrm{c}}^{0}}{N_{\mathrm{c}}^{0}} \times 100 \%$ is summarized in Fig. 7, where the interaction number at the initial time step $N_{\mathrm{c}}^{0}$ is the reference value. From this figure, it can be seen that the lattice node positions change with the compressive strain increasing, the lattice spacings become smaller and the original pore structures are compacted. Since the axial strain reaches $2 \%$, the change tends to be more obvious and the variation of compression along the $\mathrm{Y}$-axis is larger than that of the tests along $\mathrm{X}$-axis. This tendency could hint that in this digital core slice, the apertures in $Y$ direction are higher than those along the $\mathrm{X}$-axis.

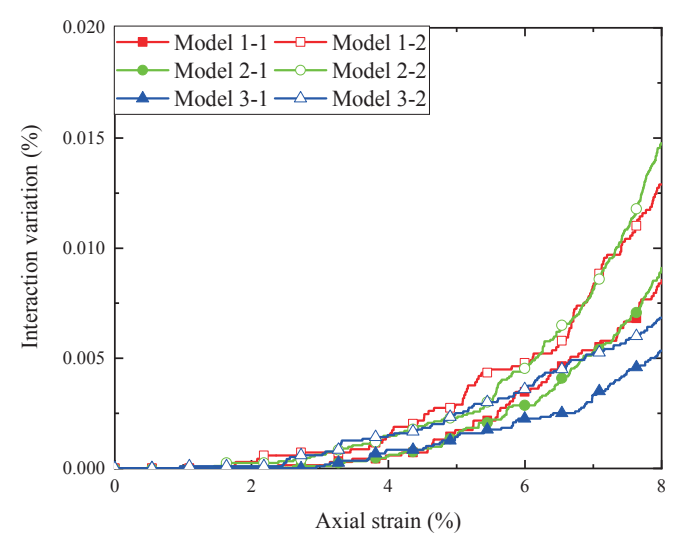

Figure 7: Interaction variation with axial strain. 


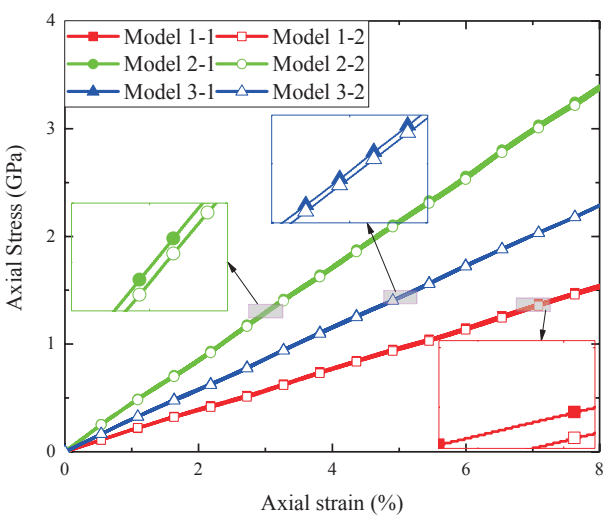

(a)

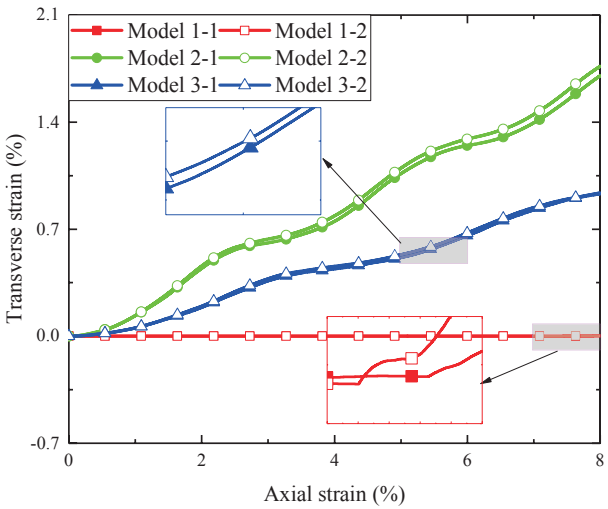

(b)

Figure 8: Elastic constant estimation: (a) for Young's moduli; (b) for Poisson's ratios.

Figs. 8 show the variations of axial stresses and transverse strains with increasing axial strains, whose ratios are the Young's modulus and Poisson's ratio, respectively. We see that for each model the two curves with the uniaxial compression along the $X$ and $Y$ directions seem to be coincident, with small differences identified by zooming in on a part of the graph. In conclusion, the Longmaxi shale core under study has a small porosity and few internal micro-cracks, and only by effective elastic constants, it may be straightforward to treat falsely the sample as isotropic medium, which might be improper.

Fig. 9 reports the theoretical prediction of bulk moduli regarding increasing porosities, with comparison to the numerical results by bounding methods. It can be noted from the figure that all the numerical values fall in the zone given by bounding methods,

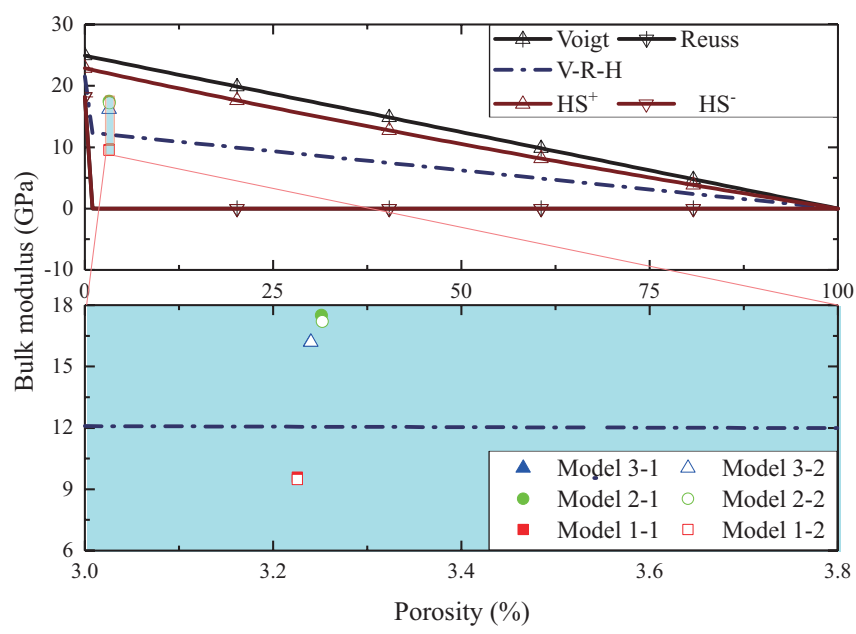

Figure 9: Bulk moduli of numerical simulations compared with bounding models. 
even though some samples behave slightly anisotropic, which violates the assumption of isotropy. It might be explained by the weakly directional preferences and the roughness of bounding methods. Particularly, the bulk moduli of Models 2 and 3 fall into the enclosed region surrounded by V-R-H average and the $\mathrm{HS}^{+}$bounds, whereas the data of Model 1 are between the V-R-H curve and the lower H-S bound, possibly because of the aforementioned calculation artifacts. In conclusion, the zone covered by V-R-H and HS ${ }^{+}$ bounds might be a better estimation of the effective elasticity for these numerical models.

\subsection{Anisotropy of LSM assemblies}

\subsubsection{Anisotropy of lattice arrangements}

In order to investigate the orientation dependence on the lattice arrangements, we emulate the homogeneous samples without micro-pores or multiphase contents, by three lattice types. To distinguish them from the LSMs of the Longmaxi shale core, they are named as Model 1*, Model 2*, and Model 3*. According to Eqs. (3.8) and (3.9), the orientation distributions with the zeroth- (black line), second- (red line), fourth- (green line), and eighth- (blue line) rank fabric tensors are calculated for the three lattice arrangements, as shown in Figs. 10. We see that the directional distributions approximated by the zeroth-rank or second-rank fabric tensors are not enough to characterize the preferred orientation. Higher-order fabric tensors, like the fourth-rank, or even the eighth-rank are more accurate. Comparison of Figs. 10(b) and 10(c) seems to indicate that nonlocal square lattices (Model 2*) are more suitable for simulating isotropic media than triangle ones (Model 3*), because all the curves of the model are nearly circles. However, from Table 4, Model $2^{*}$ has two conflicting algebraic expressions for $\lambda$. They are equivalent only if $k_{\mathrm{s}}=0$, inconsistent with the input condition in the modeling. It might illustrate that this kind of conventional fabric tensors is not a reasonable choice for the LSMs due to the absence of ample information with respect to lattice features (e.g., lattice spacing).

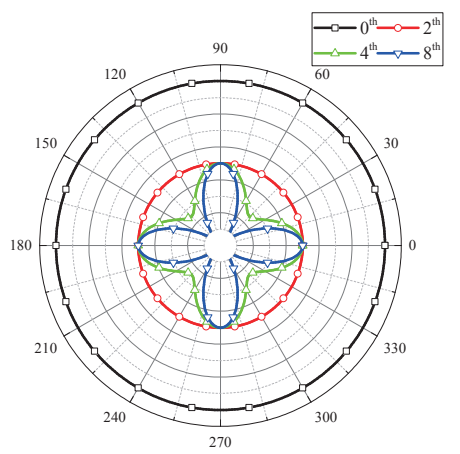

(a)

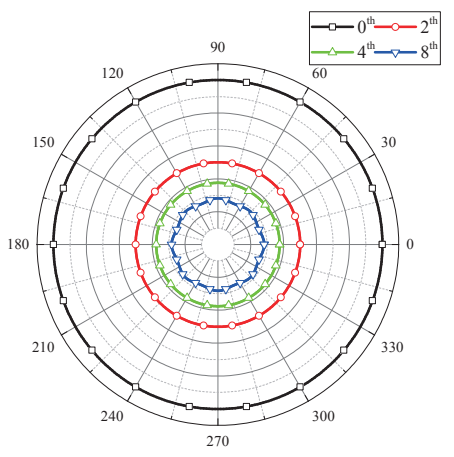

(b)

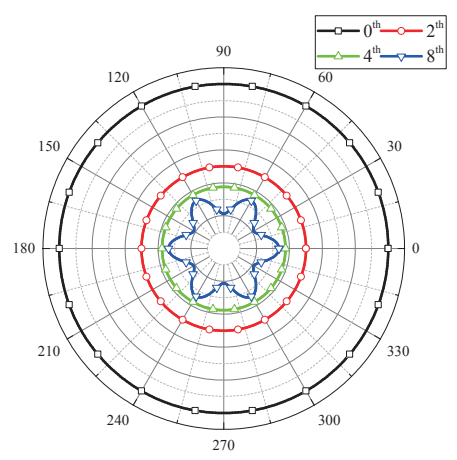

(c)

Figure 10: Polar plots for approximate orientation distributions by fabric tensors: (a) Model $1^{*}$; (b) Model 2*; (c) Model 3*. 


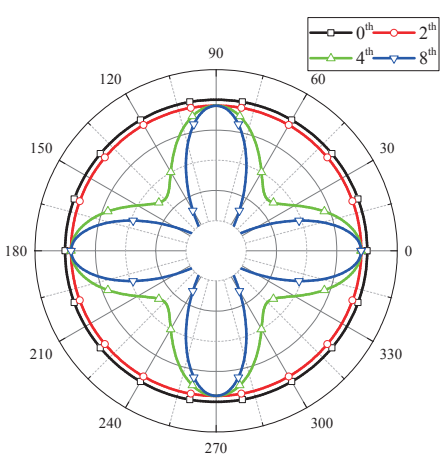

(a)

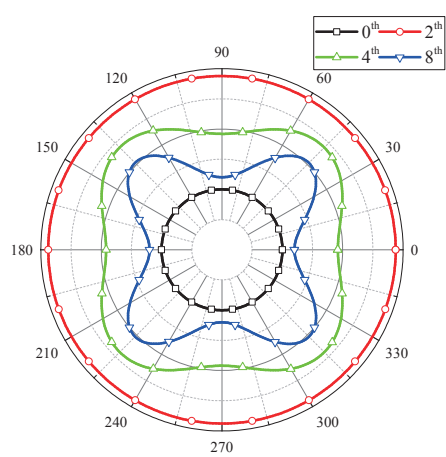

(b)

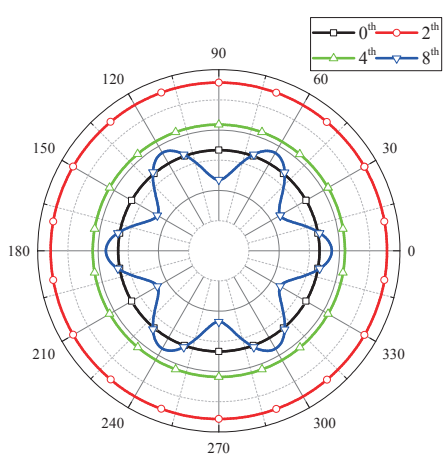

(c)

Figure 11: Polar plots for approximate orientation distributions by modified fabric tensors: (a) Model $1^{*}$; (b) Model 2*; (c) Model 3*.

By reference to damage tensors [40], we replace $\frac{1}{N_{c}}$ in Eqs. (3.8)-(3.9) by $\frac{L_{\alpha}{ }^{2}}{A}$, where $L_{\alpha}$ is the length of the $\alpha^{\text {th }}$ lattice interaction, and $A$ is the area of the numerical specimen. As demonstrated in Figs. 11, the modified fabric tensors describe well the azimuthal preference of three models in the polar coordinates. According to the approximation of higher-order fabric tensors (the eighth-ranks), we can classify these polar plots into several preferred directions: $0^{\circ}, 90^{\circ}, 180^{\circ}$ and $270^{\circ}$ for Model $1^{*}, 45^{\circ}, 135^{\circ}, 225^{\circ}$ and $315^{\circ}$ for Model $2^{*}$, and $0^{\circ}, 60^{\circ}, 120^{\circ}, 180^{\circ}, 240^{\circ}$ and $300^{\circ}$ for Model $3^{*}$. Particularly, the curves of Model 3* for the zeroth-, second-, and fourth-ranks are almost round (see Fig. 11(c)), which verifies and explains that the regular triangle lattice is an appropriate choice for modeling isotropic media. From the mathematical standpoint, there exists a formal link between the fabric tensors and the fourth-rank elasticity tensor [39]. Also, supported by Figs. 11, the fourth-rank of the modified fabric tensors seems to be an ideal candidate to characterize the biased orientations in the granular media.

\subsubsection{Anisotropy by micro-structural features}

The micro-structural features in shale cores playing an important role in the prediction of shale-gas production, particularly the void space where the natural gas are immersed. The inhomogeneity and discontinuity in the shale cores are believed to bring about local phenomena which give rise to anisotropy. In this work, since pore spaces are free of lattice nodes, the LSM numerical modeling for the digital porous core has fewer interactions of lattice nodes than those for homogeneous specimens. Figs. 12 compare the orientation distributions based on the fourth-rank of the modified fabric tensors between the digital shale core and a homogeneous specimen for the three lattice types. We see that with decreasing interactions between lattice nodes in the digital shale core, the area occupied by simulated directional plot becomes smaller, with its curve falling into the curve of the homogeneous square specimen. However, the discrepancy between them, is quite slight. According to the results, we may argue that within elastic deformation, the contributions 


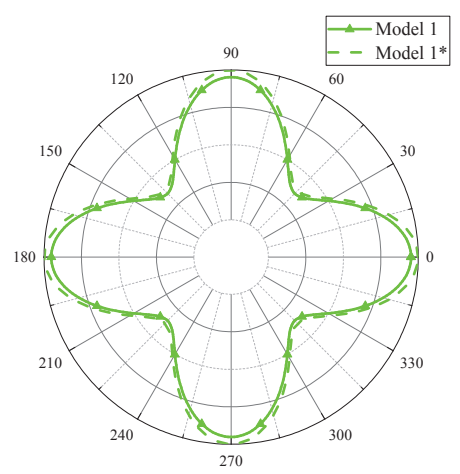

(a)

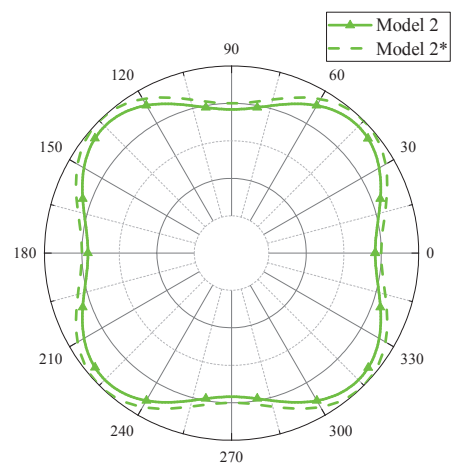

(b)

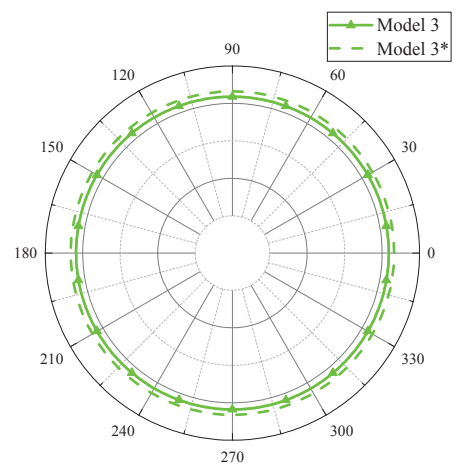

(c)

Figure 12: Comparison of the digital shale core and homogeneous square specimens based on modified fabric tensor approximation: (a) basic square lattices; (b) nonlocal square lattices; (c) regular triangle lattices.

that the void space and other micro-structural features made to the anisotropy are limited and easy to be ignored, but the discontinuities could lead to the stress concentration around them, cause an inelastic behavior, or even generate fracture networks, which will result in strong anisotropy.

\subsubsection{Stress-induced anisotropy}

This section discusses the characteristics of stress-induced anisotropy. Stresses (particularly compressive stresses) mostly tend to close micro-cracks, micro-pores, and discontinuities, or generate new fractures, thus make rock anisotropy pressure dependent [33]. Figs. 13 demonstrate how stress affects the anisotropy of three models at $\varepsilon=2.73 \%$, $\varepsilon=5.45 \%$ and $\varepsilon=8.00 \%$, which is characterized by the fourth-rank modified fabric tensors. We see that with increasing compressive strain, the statistical direction of lattice interactions is likely to be gradually perpendicular to the applied-stress axis. Holding Model 3 up as an example, in contrast to the circle curves for the unstressed homogeneous square sample (see Fig. 11(c)), the curves in Figs. 13(c) and 13(f) turn to be elliptical. As the strain becomes larger, the longer the major axis is. Only by effective mechanical parameters, this kind of azimuth variations is hardly captured, but it could pose extensive impacts on the anisotropy in mechanical behavior, failure strength, and seismic prospecting [41].

\section{Discussions}

The elastic properties of a shale core sample, like Young's modulus and Poisson's ratio, are essential mechanical parameters in the lattice spring model. These properties are decided by the normal and shear stiffness in the expressions listed in Table 4 . Theoretically, the appropriate lattice arrangement is determined for given mechanical properties. Here three lattice networks are applied, in which Models 1 and 2 could be acquired in a much easy way. However, as mentioned above that Model 1 and Model 2 are not applicable 


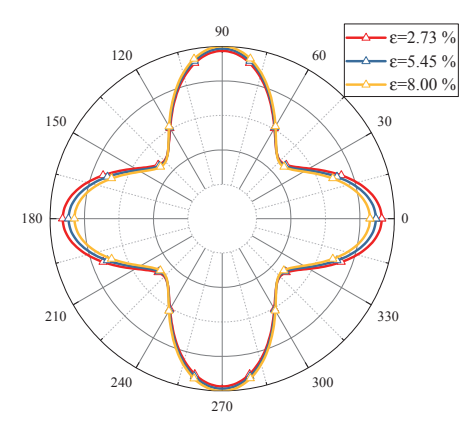

(a)

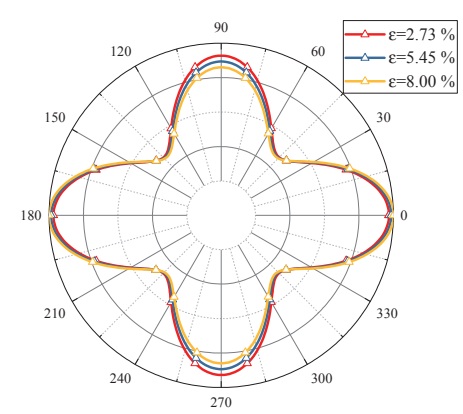

(d)

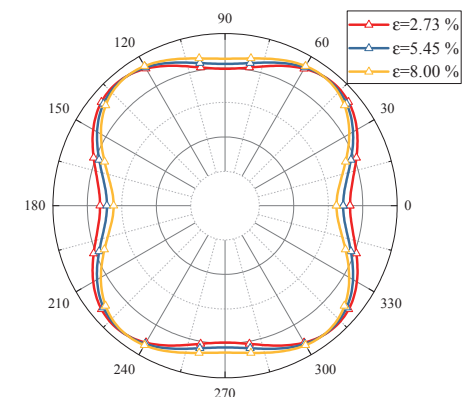

(b)

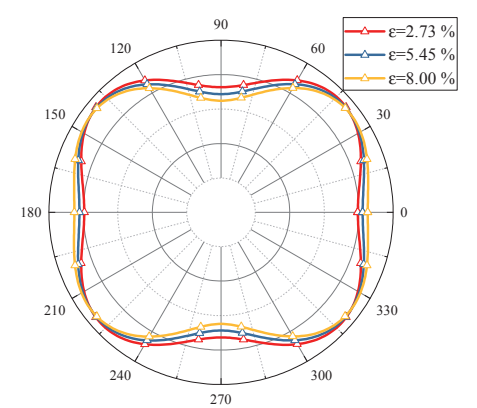

(e)

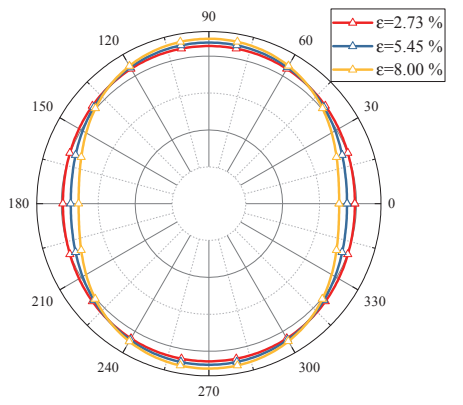

(c)

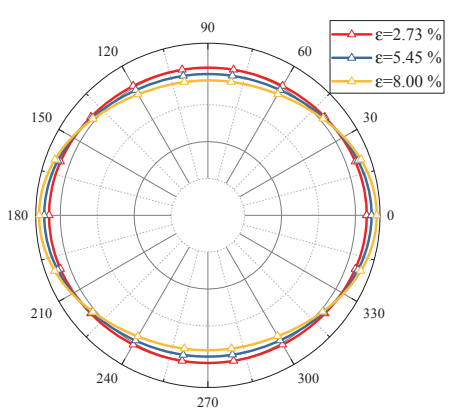

(f)

Figure 13: The approximate orientation distribution functions of fourth-rank fabric tensors at different strain levels: (a) Model 1-1; (b) Model 2-1; (c) Model 3-1; (d) Model 1-2; (e) Model 2-2; (f) Model 3-2.

to isotropic media, Jiang et al. [42] also reported that the nonlocal square lattices can't reproduce the correct elastic boundary value problems. Thereby, for this research, Model 3 seems to be a better choice and this scheme could exactly predict the mechanical behavior of rocks in the elastic deformation domain. As illustrated in Subsections 4.1 and 4.2, the elasticity acquired by the numerical simulation does agree well with the analytical prediction.

Also, the shale core used in this study might be mistakenly treated as an isotropic medium only by the effective elastic parameters calculated from compression tests along two different axes. Actually, this phenomenon based on the assumption of isotropy can't fully explain the local stress concentrations and uneven distributions of stress fields. The lattice spring model can flexibly emulate both continuous media and granular materials, but the numerical anisotropy caused by lattice arrangements may contribute to elastic anisotropy as well. As tested by numerical results, the modified fabric tensors can better characterize and quantify the anisotropy of shale cores. Within the elastic stage, for the lattice spring models, the anisotropy mainly comes from the lattice arrangements, followed by the disturbances induced by the stress fields, in which the influences caused by the micro-structures are relatively weak. It may ascribe the phenomena to the elastic assumption we employed here, lack of failure/yield criterion controlling the breakage and 
creation of the interactions and the onset and propagation of cracks, which actually are ubiquitous around the interfaces of compositions, micro-pores and micro-cracks. While, these parts are beyond the scope of this paper, and will be further discussed in future study.

Summarily, this work as the fundamental research for further exploration, concentrates on the mechanical properties in the elastic range and the main factors of reducing the numerical errors in the modeling process and quantifying the weak anisotropy of the Longmaxi shale core. The existing fractures, like bedding planes, preferred orientation or arrangement of minerals and cracks, the breakage-and-creation mechanism of the interactions between lattice nodes and the inelastic behaviors (viscous damping and temperature-dependent effect, etc.) will take into consideration in the subsequent researches. Fortunately, as for the anisotropic elasticity of the Longmaxi shale core sample, this lattice spring approach manifests its potential for reconstructing the realistic structural features with multi-scale cracks, which may help to deeper comprehend the complexity of shale and predict the shale gas plays.

\section{Conclusions}

A guideline for LSM simulations of Longmaxi shale cores via X-ray micro-CT images is present in details consistently. Distinct from the existing papers, regular triangle lattices are utilized to approximate the region of each mineral group, instead of direct meshing according to pixel coordinates. The essential characteristics regarding micro-structural features and anisotropic elasticity are investigated by emulating uniaxial compression tests. The major conclusions and merits of this scheme are organized as follows.

1. This work confirms that XMCT as a high-resolution imaging technique is suitable to capture and identify the spatial distributions and the alteration of various minerals, pores or cracks within geo-materials. Based on this approach, a comprehensive insight of the unique and complex geological features within a Longmaxi shale core could be acquired.

2. This proposed LSM scheme may be treated as a surrogate choice to numerically investigate the shale core at mesoscale in an effective way. Compared with the theoretical approximations, this algorithm could be verified and used to test the applicability of the effective medium theories.

3. Limited elastic constants (Young's moduli and Poisson's ratios along different compression axe) are insufficient to characterize the elastic properties of an anisotropic rock. Modified fabric tensors cooperating with lattice features should be introduced to quantify the direction distributions. By LSM numerical simulations, the extent of the factors that affect the anisotropy arranged in descending order could be lattice arrangements, stress field variations and micro-structural features. Additionally, basic triangle lattices are suitable to model isotropic media. 


\section{Acknowledgments}

This work is financially supported by the Strategic Priority Research Program of the Chinese Academy of Sciences, the National Natural Sciences Foundation and China Postdoctoral Science Foundation (Grant Nos. XDA14010303, XDB10010400, 41804134, and 2018M640176). We also would like to thank the editors and anonymous reviewers for insightful feedbacks.

\section{References}

[1] W.-H. Zhang, L.-Y. Fu, Y. Zhang, W.-J. Jin, Computation of elastic properties of 3D digital cores from the longmaxi shale, Applied Geophysics 13 (2016) 364-374.

[2] G. Hattori, J. Trevelyan, C. E. Augarde, W. M. Coombs, A. C. Aplin, Numerical simulation of fracking in shale rocks: Current state and future approaches, Archives of Computational Methods in Engineering 24 (2017) 281-317.

[3] C. H. Arns, M. A. Knackstedt, W. V. Pinczewski, E. J. Garboczi, Computation of linear elastic properties from microtomographic images: Methodology and agreement between theory and experiment, Geophysics 67 (2002) 1396-1405.

[4] J. Dvorkin, J. Walls, A. Tutuncu, M. Prasad, A. Nur, A. Mese, Rock property determination using digital rock physics, in: SEG Technical Program Expanded Abstracts 2003, Society of Exploration Geophysicists, 2003, pp. 1660-1663.

[5] E. Saenger, O. Krüger, S. Shapiro, Numerical considerations of fluid effects on wave propagation: Influence of the tortuosity, Geophysical Research Letters 31 (2004).

[6] X. Liu, J. Sun, H. Wang, Numerical simulation of rock electrical properties based on digital cores, Applied Geophysics 6 (2009) 1-7.

[7] W. Kewen, L. Ning, Numerical simulation of rock pore-throat structure effects on $\mathrm{NMR} \mathrm{T}_{2}$ distribution, Applied Geophysics 5 (2008) 86-91.

[8] M. R. Ceron, J. F. Martinez, E. Diaz, J. Walls, C. Sisk, Digital rock physics for reservoir characterization, in: 13th International Congress of the Brazilian Geophysical Society \& EXPOGEF, Rio de Janeiro, Brazil, 26-29 August 2013, Society of Exploration Geophysicists and Brazilian Geophysical Society, pp. 1134-1136.

[9] R. N. Vasin, H.-R. Wenk, W. Kanitpanyacharoen, S. Matthies, R. Wirth, Elastic anisotropy modeling of kimmeridge shale, Journal of Geophysical Research: Solid Earth 118 (2013) 3931-3956.

[10] S. Crampin, Seismic-wave propagation through a cracked solid: polarization as a possible dilatancy diagnostic, Geophysical Journal International 53 (1978) 467-496.

[11] S. C. Cowin, Anisotropic poroelasticity: fabric tensor formulation, Mechanics of Materials 36 (2004) 665-677.

[12] P. Hagenmuller, G. Chambon, M. Naaim, Microstructure-based modeling of snow mechanics: a discrete element approach, The Cryosphere 9 (2015) 1969-1982.

[13] Z. Gaofeng, Development of Micro-Macro Continuum-Discontinuum Coupled Numerical Method, Ph.D. thesis, ÉCOLE POLYTECHNIQUE FÉDÉRALE DE LAUSANNE, 2010.

[14] B. Harthong, L. Scholtès, F.-V. Donzé, Strength characterization of rock masses, using a coupled DEM-DFN model, Geophysical Journal International 191 (2012) 467-480. 
[15] H. Sone, M. D. Zoback, Mechanical properties of shale-gas reservoir rocks-part 2: Ductile creep, brittle strength, and their relation to the elastic modulusmechanical properties of gas shale-part 2, Geophysics 78 (2013) D393-D402.

[16] M. G. Teixeira, F. Donzé, F. Renard, H. Panahi, E. Papachristos, L. Scholtès, Microfracturing during primary migration in shales, Tectonophysics 694 (2017) 268-279.

[17] G.-F. Zhao, A. R. Russell, X. Zhao, N. Khalili, Strain rate dependency of uniaxial tensile strength in gosford sandstone by the distinct lattice spring model with X-ray micro CT, International Journal of Solids and Structures 51 (2014) 1587-1600.

[18] M. Ostoja-Starzewski, Lattice models in micromechanics, Applied Mechanics Reviews 55 (2002) 35-60.

[19] A. Hrennikoff, Solution of problems of elasticity by the framework method, J. appl. Mech. (1941).

[20] A. H. Nayfeh, M. S. Hefzy, Continuum modeling of three-dimensional truss-like space structures, AIAA Journal 16 (1978) 779-787.

[21] W. Curtin, H. Scher, Brittle fracture in disordered materials: A spring network model, Journal of Materials Research 5 (1990a) 535-553.

[22] W. Curtin, H. Scher, Mechanics modeling using a spring network, Journal of Materials Research 5 (1990b) 554-562.

[23] S. D. Saltzer, D. D. Pollard, Distinct element modeling of structures formed in sedimentary overburden by extensional reactivation of basement normal faults, Tectonics 11 (1992) 165174.

[24] P. Mora, D. Place, Numerical simulation of earthquake faults with gouge: toward a comprehensive explanation for the heat flow paradox, Journal of Geophysical Research: Solid Earth 103 (1998) 21067-21089.

[25] A. Toomey, C. J. Bean, Numerical simulation of seismic waves using a discrete particle scheme, Geophysical Journal International 141 (2000) 595-604.

[26] L. Dalguer, K. Irikura, J. Riera, H. Chiu, The importance of the dynamic source effects on strong ground motion during the 1999 Chi-Chi, taiwan, earthquake: Brief interpretation of the damage distribution on buildings, Bulletin of the Seismological Society of America 91 (2001) 1112-1127.

[27] F. Schnaid, L. d. F. Spinelli, I. Iturrioz, M. Rocha, Fracture mechanics in ground improvement design, Proceedings of the Institution of Civil Engineers-Ground Improvement 8 (2004) 715.

[28] R. Katsman, E. Aharonov, H. Scher, Numerical simulation of compaction bands in highporosity sedimentary rock, Mechanics of materials 37 (2005) 143-162.

[29] L. F. F. Miguel, J. D. Riera, I. Iturrioz, Influence of size on the constitutive equations of concrete or rock dowels, International Journal for Numerical and Analytical Methods in Geomechanics 32 (2010) 1857-1881.

[30] L. Kosteski, R. B. D'Ambra, I. Iturrioz, Fractomechanics parameter calculus using the discrete element method with bars, Latin American Journal of Solids and Structures 6 (2010) 301-321.

[31] G. F. Zhao, Developing a four-dimensional lattice spring model for mechanical responses of solids, Computer Methods in Applied Mechanics and Engineering 315 (2017) 881-895.

[32] Y. Wang, Y. Yang, T. Xiao, K. Liu, B. Clennell, G. Zhang, H. Wang, Synchrotron-based dataconstrained modeling analysis of microscopic mineral distributions in limestone, International Journal of Geosciences 4 (2013) 344.

[33] G. Mavko, T. Mukerji, J. Dvorkin, The rock physics handbook: Tools for seismic analysis of 
porous media, Cambridge university press, 2009.

[34] R. S. Carmichael, Practical Handbook of Physical Properties of Rocks and Minerals (1988), CRC press, 2017.

[35] T. Vanorio, M. Prasad, A. Nur, Elastic properties of dry clay mineral aggregates, suspensions and sandstones, Geophysical Journal International 155 (2003) 319-326.

[36] S.-F. Zhao, G.-F. Zhao, Implementation of a high order lattice spring model for elasticity, International Journal of Solids and Structures 49 (2012) 2568-2581.

[37] N. Liu, M. Li, W. Chen, Mechanical deterioration of rock salt at different confinement levels: A grain-based lattice scheme assessment, Computers and Geotechnics 84 (2017) 210-224.

[38] K. Ken-Ichi, Distribution of directional data and fabric tensors, International Journal of Engineering Science 22 (1984) 149-164.

[39] P. Zysset, A. Curnier, An alternative model for anisotropic elasticity based on fabric tensors, Mechanics of Materials 21 (1995) 243-250.

[40] G. Z. Voyiadjis, P. I. Kattan, Damage mechanics with fabric tensors, Mechanics of Advanced Materials and Structures 13 (2006) 285-301.

[41] K. Bandyopadhyay, Seismic anisotropy: Geological causes and its implications to reservoir geophysics, Stanford University, 2009.

[42] C. Jiang, G.-F. Zhao, Implementation of a coupled plastic damage distinct lattice spring model for dynamic crack propagation in geomaterials, International Journal for Numerical and Analytical Methods in Geomechanics 42 (2018) 674-693. 